\title{
Fasiglifam/TAK-875, a Selective GPR40 Agonist, Improves Hyperglycemia in Rats Unresponsive to Sulfonylureas and Acts Additively with Sulfonylureas $\$$
}

\author{
Ryo Ito, Yoshiyuki Tsujihata, Masami Suzuki, Kazumasa Miyawaki, Kae Matsuda, \\ and Koji Takeuchi \\ Cardiovascular and Metabolic Drug Discovery Unit, Pharmaceutical Research Division, Takeda Pharmaceutical Company \\ Limited, Kanagawa, Japan
}

Received November 25, 2015; accepted January 21, 2016

\begin{abstract}
Sulfonylureas (SUs) are widely used insulin secretagogues, but they have adverse effects including hypoglycemia and secondary failure. Fasiglifam/TAK-875, a selective GPR40 agonist, enhances glucose-stimulated insulin secretion and improves hyperglycemia. In the present study, we compared the in vivo glucoselowering effects of fasiglifam with SUs. The risk of secondary failure of fasiglifam and the efficacy in rats desensitized to SUs were also evaluated. Moreover, we assessed whether fasiglifam was effective when combined with SUs. In diabetic neonatally streptozotocin-induced rats 1.5 days after birth (N-STZ-1.5), oral administrations of fasiglifam $(3-30 \mathrm{mg} / \mathrm{kg}$ ) dose dependently improved glucose tolerance; the effect was greater than that of glibenclamide at maximal effective doses (glucose AUC: fasiglifam, $-37.6 \%$; glibenclamide, $-12.3 \%)$. Although the glucoselowering effects of glibenclamide $(10 \mathrm{mg} / \mathrm{kg} /$ day $)$ were completely
\end{abstract}

diminished in N-STZ-1.5 rats after 4 weeks of treatment, effects were maintained in rats receiving fasiglifam (10 mg/kg/day), even after 15 weeks. Fasiglifam (3-10 mg/kg) was still effective in two models desensitized to SUs: 15-week glibenclamide-treated $\mathrm{N}-\mathrm{STZ}-1.5$ rats and aged Zucker diabetic fatty (ZDF) rats. Acute administration of fasiglifam $(3 \mathrm{mg} / \mathrm{kg})$ and glimepiride $(10 \mathrm{mg} / \mathrm{kg})$ in combination additively decreased glucose AUC (fasiglifam, $-25.3 \%$; glimepiride, $-20.0 \%$; combination, $-43.1 \%)$. Although glimepiride $(10 \mathrm{mg} / \mathrm{kg})$ decreased plasma glucose below normal in nonfasted control rats, fasiglifam $(3 \mathrm{mg} / \mathrm{kg}$ ) maintained normoglycemia, and no further exaggeration of hypoglycemia was observed with combination treatment. These results indicate that GPR40 agonists could be more effective and durable than SUs. Our results also provide new insights into GPR40 pharmacology and rationale for the use of GPR40 agonists in diabetic patients with SU failure.

\section{Introduction}

The loss of glucose-dependent insulin secretion from pancreatic $\beta$-cells is responsible for the onset and progression of type 2 diabetes (Leahy, 2005; Wajchenberg, 2007). Oral agents that stimulate insulin secretion such as sulfonylureas (SUs) have been widely used for the treatment of type 2 diabetes therapy for nearly 30 years (Rendell, 2004). These agents stimulate insulin secretion by closing ATP-sensitive $\mathrm{K}^{+}$ channels on $\beta$-cells, leading to depolarization and subsequent increase in intracellular $\mathrm{Ca}^{2+}$. However, SUs act on $\beta$-cells to continuously secrete insulin independent of blood glucose levels, which may lead to hypoglycemia. In addition, after long-term SU therapy, a number of patients begin to show an

Parts of these studies were presented at the following workshop: Ito $R$ Tsujihata Y, Suzuki M, Matsuda K, Miyawaki K, Negoro N, and Takeuchi K (2012) TAK-875, a GPR40 agonist, improves postprandial hyperglycemia additively with sulfonylurea and is effective in rats with sulfonylurea failure. 72nd Scientific Sessions of the American Diabetes Association; 2012 Jun 8-12; Philadelphia, PA.

dx.doi.org/10.1124/jpet.115.230730.

S This article has supplemental material available at jpet.aspetjournals. org. increase in blood glucose levels, necessitating the addition of other drugs with a different mechanism of action (Matthews et al., 1998). This clinical phenomenon has been termed secondary failure of SUs. This is thought to be broadly associated with $\beta$-cell exhaustion by long-term overstimulation and glucose toxicity caused by poor glycemic control (Poitout and Robertson, 2002; Rustenbeck, 2002). Therefore, there is a need for agents that induce glucose-dependent insulin secretion through new mechanisms of action, with low risk of the adverse effects associated with SU treatment.

GPR40/FFAR1 is a G-protein-coupled receptor highly expressed in pancreatic $\beta$-cells (Briscoe et al., 2003; Itoh et al., 2003; Kotarsky et al., 2003). Long- and medium-chain free fatty acids (FFAs) are endogenous ligands for GPR40, and the activation of GPR40 by FFAs leads to augmentation of insulin secretion through activation of $\mathrm{G} \alpha \mathrm{q}$, which promotes phospholipase C-mediated production of inositol 1,4,5triphosphate, thereby resulting in augmentation of intracellular $\mathrm{Ca}^{2+}$ (Fujiwara et al., 2005; Shapiro et al., 2005; Feng et al., 2006; Sakuma et al., 2015). This mechanism is novel and is clearly distinct from other oral insulinotropic drugs, such as

ABBREVIATIONS: ANOVA, analysis of variance; AUC, area under the curve; DPP-4, dipeptidyl peptidase-4; FFAs, free fatty acids; N-STZ 1.5, neonatally streptozotocin-induced 1.5 days after birth; STZ, streptozotocin; SUs, sulfonylureas; WKY, Wistar Kyoto; ZDF, Zucker diabetic fatty; ZL, Zucker lean. 
SUs (Rendell, 2004) and dipeptidyl peptidase-4 (DPP-4) inhibitors (Drucker, 2007).

Fasiglifam is a selective and orally available GPR40 agonist that improves postprandial and fasting hyperglycemia by potentiating insulin secretion in diabetic rats (Tsujihata et al., 2011; Negoro et al., 2012; Ito et al., 2013). A recent in vitro study has shown that fasiglifam acts as an ago-allosteric modulator of GPR40 and exerts the effects cooperatively with endogenous ligand FFAs rather than competing with one another (Yabuki et al., 2013). The most clinically meaningful feature of GPR40 agonists is the increase in insulin secretion solely in the presence of high glucose concentration, in contrast to SUs, which enhance insulin release regardless of glucose concentration (Tsujihata et al., 2011). This glucosedependent insulin secretion likely leads not only to a low risk of hypoglycemia, but also to a lesser probability of $\beta$-cell exhaustion due to mitigating the burden of $\beta$-cells through appropriate insulin secretion compared with SUs. However, the detailed comparison of in vivo acute and chronic profiles between GPR40 agonists and SUs has not been sufficiently studied.

Because combination therapy with antidiabetic drugs with different mechanism of action is often used (Charpentier, 2002), information on the combinational effects of existing drugs is important. Fasiglifam has previously shown additive glycemic control in diabetic rodents in combination with the peripheral insulin sensitizer metformin (Ito et al., 2013). Interestingly, it has been reported that the glucose dependency of GPR40-mediated insulin secretion is uncoupled by the presence of SUs in rat insulinoma cells (Yang et al., 2010). This implies that GPR40 agonists would enhance insulin secretion even at low plasma glucose levels when administered with SUs, thereby promoting hypoglycemia. From the aspect of safety, as well as the mechanism of underlying insulinotropic action, there is interest in determining whether GPR40 agonists can work cooperatively with SUs.

Fasiglifam is the first GPR40 agonist to significantly improve glycemic control in patients with type 2 diabetes (Kaku et al., 2015). However, the clinical development of fasiglifam was terminated because of concerns about liver safety. Other than fasiglifam, several other GPR40 agonists are currently still in the preclinical and clinical stages of development (Mancini and Poitout, 2015). Given that GPR40 agonists potentially constitute a new class of insulinotropic drugs, further understanding of their differences from and interactions with widely used insulinotropic drugs is necessary. In the present study, we analyzed the acute glucoselowering effects between fasiglifam and SUs and assessed the risk of secondary failure of fasiglifam in type 2 diabetic rats. We also examined whether treatment with fasiglifam lowers glucose in two rat models desensitized to SU treatment: 15week glibenclamide-treated neonatally streptozotocin-induced rats 1.5 days after their birth (N-STZ-1.5) and aged Zucker diabetic fatty (ZDF) rats. Finally, we evaluated the effects of fasiglifam in combination with SUs on postprandial hyperglycemia and the risk of hypoglycemia.

\section{Materials and Methods}

Animals. Male N-STZ-1.5 rats were prepared by administering $120 \mathrm{mg} / \mathrm{kg}$ of streptozotocin (STZ) subcutaneously to male Wistar Kyoto (WKY) rats on day 1.5 after birth (Portha et al., 1989). The N-STZ-1.5 rats and neonatal saline-injected WKY rats (non-diabetic control rats) were obtained from Takeda Rabics Limited (Osaka, Japan). Male ZDF (leprfa/CrlCrlj fatty fa/fa) rats and their nondiabetic littermates, male Zucker lean (ZL) (leprfa/CrlCrlj lean $+/+$ or fa/+) rats, were obtained from Charles River Laboratories Japan, Inc (Yokohama, Japan). Male Sprague-Dawley rats were purchased from Clea, Japan, Inc. (Tokyo, Japan). All rats were housed in individual metal cages in a room with controlled temperature $\left(23^{\circ} \mathrm{C}\right)$, humidity $(55 \%)$, and 12 hours dark/12 hours light. Rats were allowed free access to powdered standard laboratory chow diet (CE-2, Clea, Japan, Inc.) and tap water. The care and use of the animals and experimental protocols used in this study were approved by the Experimental Animal Care and Use Committee of Takeda Pharmaceutical Company Limited (Kanagawa, Japan).

Materials. Fasiglifam ([(3S)-6-(\{2',6'-dimethyl-4'-[3-(methylsulfonyl) propoxy]biphenyl-3-yl\}methoxy)-2,3-dihydro-1-benzofuran-3-yl]acetic acid hemihydrate) and alogliptin (Feng et al., 2007) were synthesized at Chemical Development Laboratories, Takeda Pharmaceutical Company Limited. Glibenclamide and glimepiride were purchased from Wako Pure Chemical Industries, Limited (Osaka, Japan). Drugs were suspended in $0.5 \%$ methylcellulose solution (Wako Pure Chemical Industries, Limited).

Oral Glucose Tolerance Test (Mono-treatment). At the age of 20 to 27 weeks, N-STZ-1.5 and WKY rats underwent overnight fasting. N-STZ-1.5 rats were divided into 7 groups $(n=6)$ so that plasma levels of glucose, triglycerides, and body weight were not statistically significantly different among groups. Each group of N-STZ-1.5 rats was given vehicle $(0.5 \%$ methylcellulose $)$, fasiglifam $(3,10$, and $30 \mathrm{mg} / \mathrm{kg})$, or glibenclamide $(3,10$, and $30 \mathrm{mg} / \mathrm{kg})$ orally in a volume of $5 \mathrm{ml} / \mathrm{kg}$. WKY rats $(n=5)$ were given vehicle orally. Plasma parameters measured for the grouping of the animals were used as baseline data. Sixty minutes after drug administration, all animals received an oral glucose load $(1.5 \mathrm{~g} / \mathrm{kg})$. Blood samples were collected from the tail vein just before (time 0 ) and 10, 30, 60, and 120 minutes after glucose load for the determination of plasma glucose and insulin levels.

Evaluation of the Risk of Secondary Failure of Fasiglifam. N-STZ-1.5 rats at 16 weeks of age were divided into 2 groups $(n=12)$ so that plasma levels of glucose, triglycerides, total cholesterol, nonesterified fatty acids, and body weight were not statistically significantly different among groups. Each group was given vehicle or fasiglifam $(10 \mathrm{mg} / \mathrm{kg})$ orally in a volume of $2.5 \mathrm{ml} / \mathrm{kg}$ once daily for 15 weeks. On day 90 , one rat in the vehicle-treated group died (unexpected decrease in body weight); thus, this rat was excluded from data analysis. After 14 weeks of treatment, each group was regrouped into 2 groups ( $n=5$ or 6 ). After an additional week of treatment, each group underwent overnight fasting and received vehicle or fasiglifam $(3 \mathrm{mg} / \mathrm{kg})$ orally in a volume of $2.5 \mathrm{ml} / \mathrm{kg}$ 30 minutes before glucose load $(1 \mathrm{~g} / \mathrm{kg})$. Blood samples were collected from the tail vein just before (time 0 ) and 10, 30, 60, and 120 minutes after glucose load for the determination of plasma glucose and insulin levels. WKY rats $(n=6)$ were similarly treated with vehicle for the same period and underwent an oral glucose tolerance test. Rats were killed by exsanguination, and the pancreas was isolated for immunostaining. Isolated pancreata were fixed overnight with Bouin's solution (Polysciences, Inc., Warrington, PA) and transferred into 70\% ethanol before processing through paraffin. Immunostaining with antiinsulin and anti-glucagon antibodies (DAKO, Glostrup, Denmark) were performed, and images were obtained with an inverted microscope IX71 (Olympus, Tokyo, Japan).

Evaluation of Rats with SU Secondary Failure. N-STZ-1.5 rats (20 weeks of age) were divided into 2 groups ( $n=16$ and $n=24$ ) so that plasma levels of glucose and body weight were not statistically significantly different among groups. Each group was given vehicle $(n=16)$ or glibenclamide ( $10 \mathrm{mg} / \mathrm{kg}, n=24)$ orally in a volume of $2.5 \mathrm{ml} / \mathrm{kg}$ once daily for 4 weeks. After overnight fasting, the repeated vehicle-treated group was regrouped into 2 groups, whereas the glibenclamide-treated group was regrouped into 3 groups $(n=8)$. The repeated vehicle-treated groups were given vehicle or glibenclamide $(10 \mathrm{mg} / \mathrm{kg}$ ) orally in a volume of $5 \mathrm{ml} / \mathrm{kg} 1$ hour before 
A

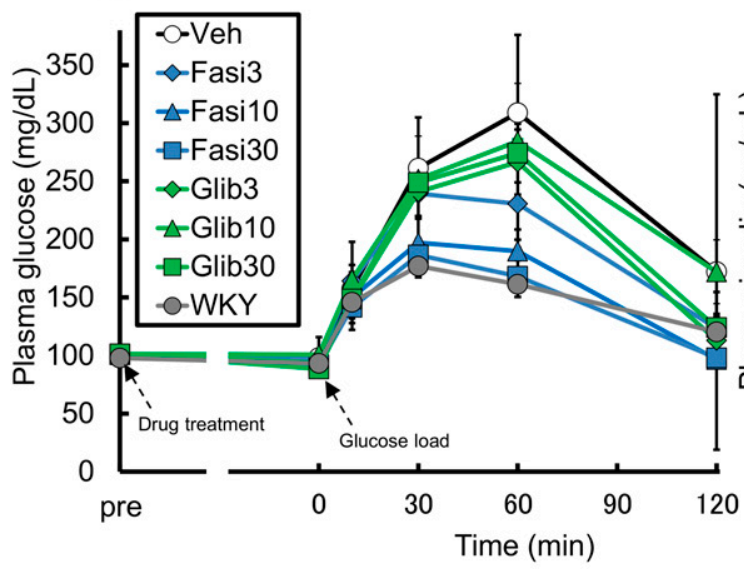

C

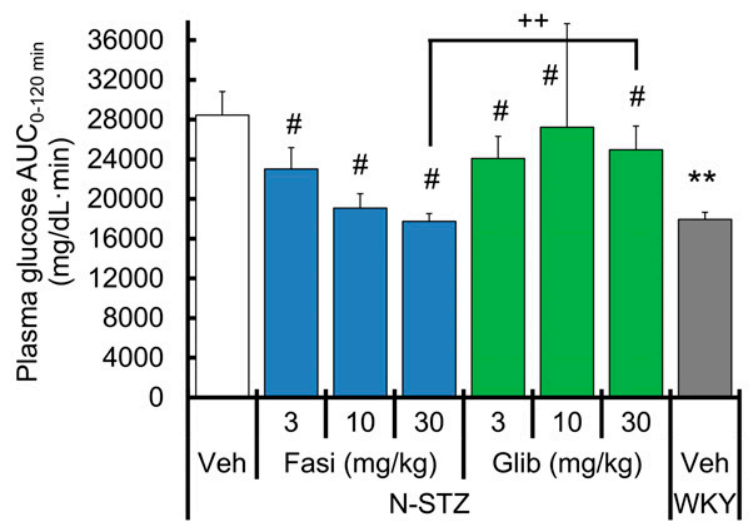

B

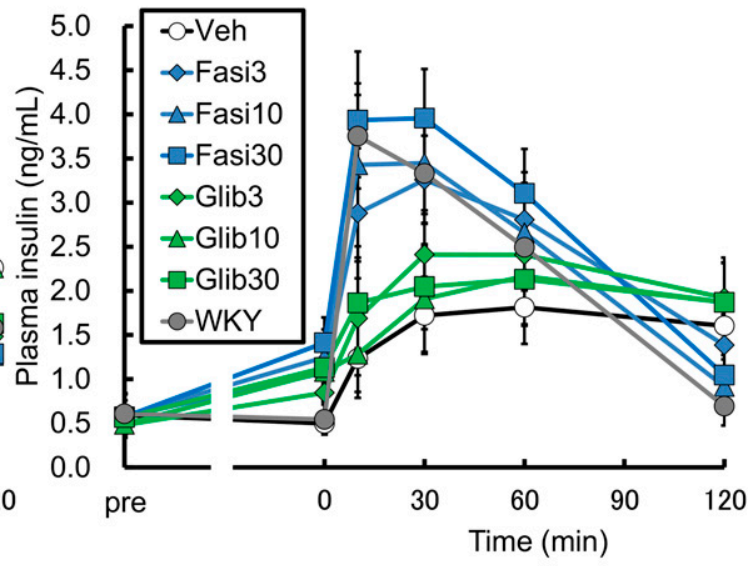

D

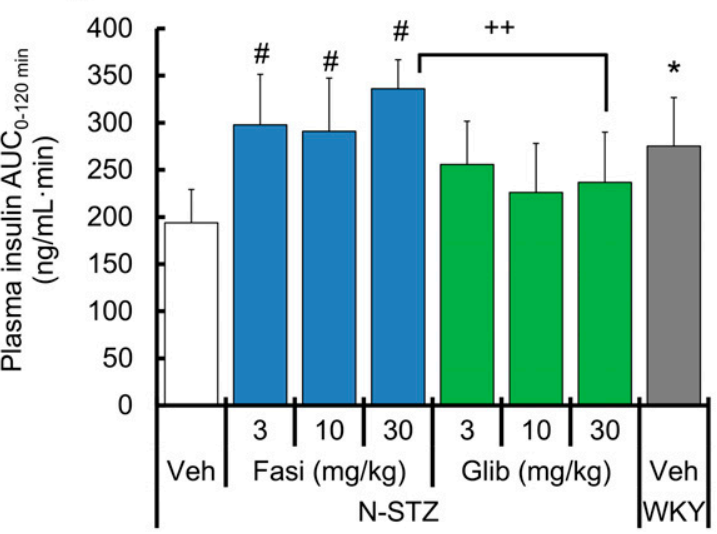

Fig. 1. Fasiglifam improves glucose tolerance more effectively than glibenclamide during oral glucose tolerance tests in male N-STZ-1.5 rats. (A) and (B) Time-dependent changes in plasma glucose and insulin levels, respectively, after glucose load (1.5 g/kg) in N-STZ-1.5 rats treated with vehicle (Veh), $3-30 \mathrm{mg} / \mathrm{kg}$ of fasiglifam (Fasi), or 3-30 mg/kg of glibenclamide (Glib) and in WKY rats (normal controls) treated with vehicle. Data in (C) and (D) represent plasma glucose $\mathrm{AUC}_{0-120}$ minutes and plasma insulin $\mathrm{AUC}_{0-120}$ minutes, respectively. ${ }^{\#} P \leq 0.025$ compared with vehicle-treated N-STZ-1.5 rats by one-tailed Williams' test or one-tailed Shirley-Williams test. $* P \leq 0.05, * * P \leq 0.01$ compared with vehicle-treated N-STZ-1.5 rats by Student's $t$ test or Aspin-Welch test. ${ }^{++} P \leq 0.01$ by Student's $t$ test or Aspin-Welch test. Values are mean \pm S.D. ( $n=6$ for N-STZ-1.5 rats, $n=5$ for WKY rats).

oral glucose load ( $1 \mathrm{~g} / \mathrm{kg}$ ). The repeated glibenclamide-treated groups were given vehicle, glibenclamide $(10 \mathrm{mg} / \mathrm{kg})$, or fasiglifam $(3 \mathrm{mg} / \mathrm{kg})$ orally in a volume of $5 \mathrm{ml} / \mathrm{kg} 1$ hour before oral glucose load. Blood samples were collected from the tail vein just before (time 0 ) and 10 , 30,60 , and 120 minutes after oral glucose load for the determination of plasma glucose and insulin levels.

Effects on Fasting Hyperglycemia in Severe Diabetic Rats. At the age of 15 weeks of age, male ZDF and ZL rats underwent overnight fasting. ZDF rat were divided into 3 groups $(n=6)$ so that plasma levels of glucose, triglycerides, and body weight were not statistically significantly different among groups. Each group of ZDF rats was given vehicle, fasiglifam $(10 \mathrm{mg} / \mathrm{kg})$, or glimepiride $(10 \mathrm{mg} / \mathrm{kg})$ orally in a volume of $5 \mathrm{ml} / \mathrm{kg}$. ZL rats $(n=6)$ were given vehicle orally. Plasma glucose and insulin levels were determined using blood samples collected from the tail vein at $0,30,60,120,240$, and 360 minutes after drug administration.

Oral Glucose Tolerance Test (Combination Treatment). At the age of 21 weeks, N-STZ-1.5 and WKY rats underwent overnight fasting. N-STZ-1.5 rats were divided into 4 groups $(n=6)$ so that plasma levels of glucose, triglycerides, and body weight were not statistically significantly different among groups. Each group was given vehicle, fasiglifam (3 $\mathrm{mg} / \mathrm{kg})$, glimepiride $(10 \mathrm{mg} / \mathrm{kg})$, or fasiglifam $(3 \mathrm{mg} / \mathrm{kg})$ in combination with glimepiride $(10 \mathrm{mg} / \mathrm{kg})$ orally in a volume of $5 \mathrm{ml} / \mathrm{kg}$. WKY rats $(n=5)$ were given vehicle orally. Sixty minutes after drug administration, all animals received an oral glucose load $(1.5 \mathrm{~g} / \mathrm{kg})$. Blood samples were collected from the tail vein just before glucose load (time 0 ) and 10,30,60, and 120 minutes after glucose load for the determination of plasma glucose and insulin levels.

Evaluation of Hypoglycemia Risk. At the age of 10 weeks, nonfasted male Sprague-Dawley rats were divided into 6 groups $(n=6)$ so that body weight was not statistically significantly different among groups. Each group was given vehicle, fasiglifam ( $3 \mathrm{mg} / \mathrm{kg})$, alogliptin $(1 \mathrm{mg} / \mathrm{kg})$, glimepiride $(10 \mathrm{mg} / \mathrm{kg})$, fasiglifam $(3 \mathrm{mg} / \mathrm{kg})$ in combination with glimepiride $(10 \mathrm{mg} / \mathrm{kg})$, or alogliptin $(1 \mathrm{mg} / \mathrm{kg})$ in combination with glimepiride $(10 \mathrm{mg} / \mathrm{kg}$ ) orally in a volume of $5 \mathrm{ml} / \mathrm{kg}$. Plasma glucose and insulin levels were determined using blood samples collected from the tail vein just before drug treatment (time 0) and $0.5,1,2,4$, and 6 hours after drug administration.

Measurements of Plasma Parameters. Plasma levels of glucose, triglycerides, total cholesterol, and nonesterified fatty acids were enzymatically measured by Autoanalyzer 7080 (Hitachi, Tokyo, Japan). Plasma insulin was measured using radioimmunoassay kit (Millipore, Bedford, MA). For determination of low levels of plasma insulin, rat insulin enzyme-linked immunosorbent assay kits (Morinaga Institute of Biologic Science, Yokohama, Japan) were used.

Statistical Analysis. To evaluate effects of drugs during oral glucose tolerance test, total plasma glucose area under the curve (AUC) and plasma insulin AUC were calculated. For evaluation of effects under fasted and nonfasted state, change in plasma glucose and change in plasma insulin levels were calculated from the difference between values at indicated time point and initial value, and change in 

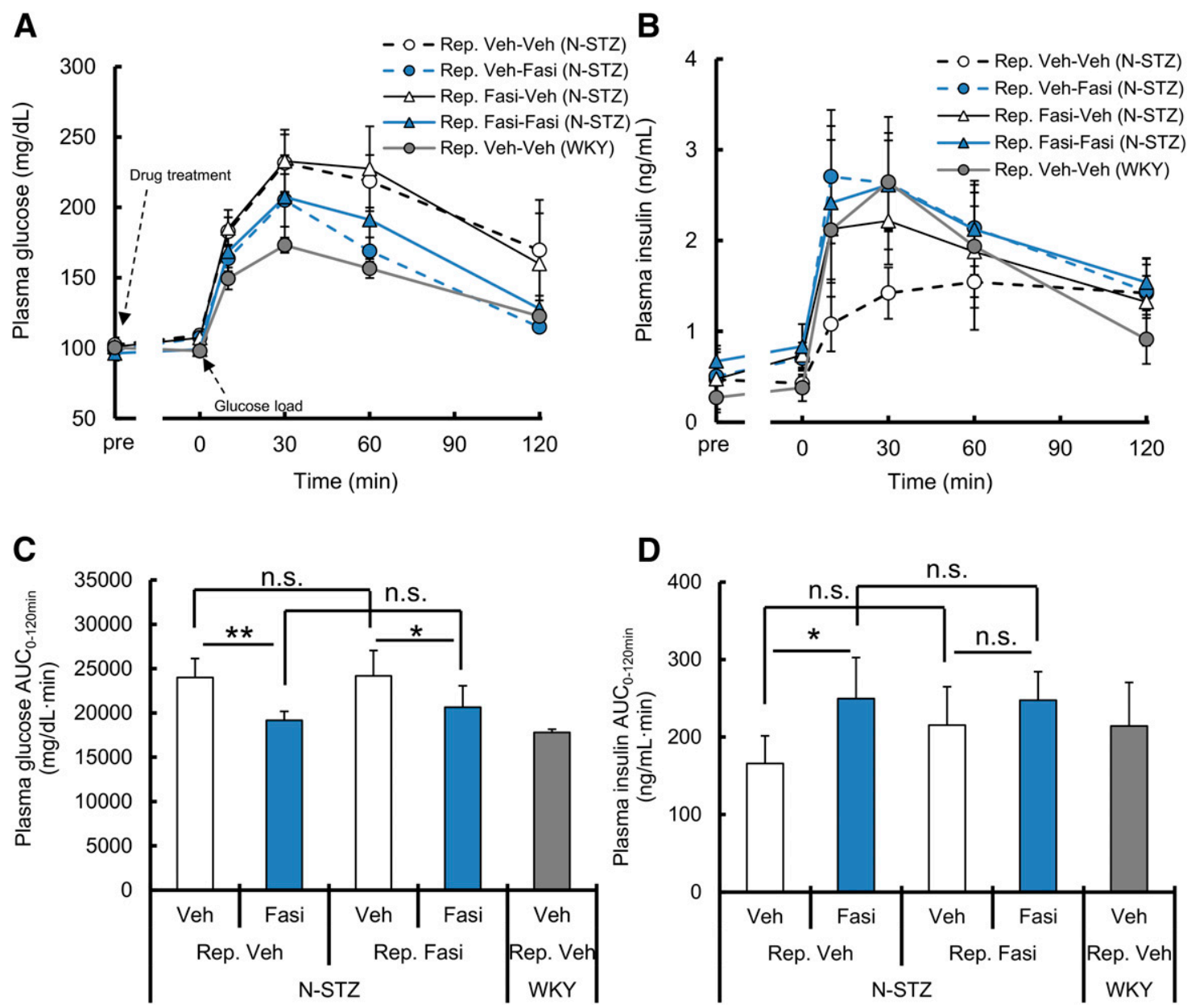

Fig. 2. Chronic treatment with fasiglifam does not cause secondary loss of glucose-lowering efficacy. N-STZ-1.5 rats were treated once daily with vehicle (Rep. Veh) or fasiglifam (Rep. Fasi, $10 \mathrm{mg} / \mathrm{kg}$ ) for 15 weeks. After fasting overnight, vehicle (Veh) or fasiglifam (Fasi, $3 \mathrm{mg} / \mathrm{kg}$ ) was given orally to both groups 30 minutes before glucose load (1 g/kg). Similarly, WKY rats (normal controls) were treated with vehicle once daily for 15 weeks, and the same oral glucose tolerance test was performed. (A) and (B) Time-dependent changes in plasma glucose and insulin levels, respectively. Data in (C) and (D) represent plasma glucose $\mathrm{AUC}_{0-120}$ minutes and plasma insulin $\mathrm{AUC}_{0-120}$ minutes, respectively. ${ }^{*} P \leq 0.05,{ }^{* *} P \leq 0.01$ by Student's $t$ test or Aspin-Welch test. n.s., Not significant. Values are mean \pm S.D. $(n=5$ or 6$)$.

plasma glucose AUC and change in plasma insulin AUC were calculated using these values. Statistical differences between 2 groups were analyzed using the Student's $t$ test or the Aspin-Welch test depending on variances between groups. Dose-dependent effects were analyzed using a one-tailed Williams' test or a one-tailed ShirleyWilliams test depending on variances between groups. Combination effects were assessed using a two-way analysis of variance (ANOVA) (Moritoh et al., 2009). The results were interpreted as follow: i) when a significant interaction effect $(P \leq 0.05)$ was observed, the effect of the combination was considered to be synergistic (when the effect of the combination treatment exceeds the sum of the effect of the monotreatment) and ii) when no significant interaction and significant main effects of both drugs $(P \leq 0.05)$ were observed, the effect was considered to be additive (when the effect of the combination treatment equals the sum of the effect of the monotreatment). All data are presented as mean \pm S.D.

\section{Results}

Fasiglifam Improves Postprandial Hyperglycemia More Effectively than Glibenclamide. The glucoselowering effects and insulinotropic action of fasiglifam and SUs were first compared by an oral glucose tolerance test in type 2 diabetic N-STZ-1.5 rats. Compared with vehicle, oral administration of fasiglifam at doses of 3,10 , and $30 \mathrm{mg} / \mathrm{kg}$ dose dependently reduced plasma glucose AUC by 19.1, 33.0, and $37.6 \%$, respectively, and the glucose tolerance in rats given fasiglifam $(30 \mathrm{mg} / \mathrm{kg})$ reached a level comparable to that in normal WKY rats (Fig. 1, A and C). Consistent with glucoselowering effects, fasiglifam at doses of 3,10 , and $30 \mathrm{mg} / \mathrm{kg}$ robustly increased plasma insulin AUC 1.5-, 1.5-, and 1.7-fold, respectively (Fig. 1, B and D). Conversely, compared with vehicle, 3,10 , and $30 \mathrm{mg} / \mathrm{kg}$ of glibenclamide significantly decreased plasma glucose AUC by 15.3, 4.3, and 12.3\%, respectively; the effects reached a plateau at a dose of $3 \mathrm{mg} / \mathrm{kg}$ (Fig. 1, A and C). Glibenclamide showed a trend toward increase in plasma insulin AUC after glucose load (Fig. 1, B and D). The similar glucose-lowering effects and insulinotropic action were also observed with glimepiride (3-30 mg/kg; (Supplemental Fig. 1). When compared at a maximal dose of $30 \mathrm{mg} / \mathrm{kg}$, the improvement in glucose tolerance and insulin secretion in response to glucose in diabetic rats treated with fasiglifam was significantly greater than those treated with glibenclamide (Fig. 1, C and D). At this dose, both fasiglifam and glibenclamide decreased fasting plasma glucose and increased fasting plasma insulin levels just before glucose load (time 0); however, between drugs, 


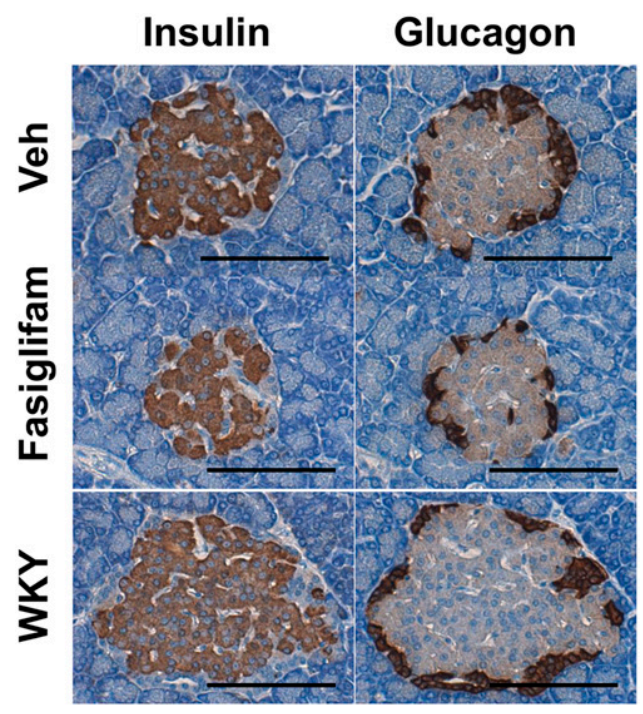

Fig. 3. Pancreatic islet morphology in male N-STZ-1.5 rats after multiple dosing of fasiglifam. N-STZ-1.5 rats were treated with vehicle (Veh) or fasiglifam $(10 \mathrm{mg} / \mathrm{kg})$, and WKY rats (normal controls) were treated with vehicle, once daily for 15 weeks. Fixed pancreata were immunostained with anti-insulin and antiglucagon antibodies. Scale bar $=100 \mu \mathrm{m}$.

these effects were not significantly different (fasting plasma glucose: vehicle, $98.1 \pm 2.1 \mathrm{mg} / \mathrm{dl}$; fasiglifam, $91.9 \pm 4.1 \mathrm{mg} / \mathrm{dl}$; glibenclamide, $88.4 \pm 3.7 \mathrm{mg} / \mathrm{dl}$; fasting plasma insulin: vehicle, $0.5 \pm 0.1 \mathrm{ng} / \mathrm{ml}$; fasiglifam, $1.4 \pm 0.3 \mathrm{ng} / \mathrm{ml}$; glibenclamide, $1.1 \pm 0.2 \mathrm{ng} / \mathrm{ml}$; Fig. 1 , A and B).

Chronic Treatment with Fasiglifam Does Not Cause Secondary Loss of Glucose-lowering Efficacy. It was previously reported that 4-week treatment with glibenclamide induces the exacerbation of glucose tolerance and the loss of response to glibenclamide in N-STZ-1.5 rats, leading to the development of secondary failure (Asakawa et al., 2009). To determine if chronic treatment with fasiglifam results in the similar secondary loss of its glucose-lowering efficacy, an oral glucose tolerance test was performed in N-STZ-1.5 rats after long-term multiple doses of fasiglifam. Based on the pharmacokinetic profiles in rats, 3 to $10 \mathrm{mg} / \mathrm{kg}$ of fasiglifam was selected in the following study; this dose is close to the clinical doses of fasiglifam in humans (Negoro et al., 2010; Naik et al., 2012). After 14 weeks of treatment (1 week before an oral glucose tolerance test), no significant effects on changes in body weight were observed between vehicle and fasiglifam groups (changes in body weight from initial values: vehicle, $71.3 \pm 4.8 \mathrm{~g}$; fasiglifam, $68.4 \pm 5.7 \mathrm{~g}$ ). In the N-STZ-1.5 rats given fasiglifam $(10 \mathrm{mg} / \mathrm{kg})$ for 15 weeks, glucose tolerance and insulin secretion after glucose load were not significantly different compared with the rats given vehicle for 15 weeks [Fig. 2; repeatedly treated (Rep). Veh-Veh versus Rep. FasiVeh]. Moreover, acute administration of fasiglifam $(3 \mathrm{mg} / \mathrm{kg})$ 30 minutes before glucose load significantly decreased glucose excursion in rats pretreated with fasiglifam (Fig. 2. A and C; Rep. Fasi-Veh versus Rep. Fasi-Fasi), and the glucoselowering effects were comparable to that seen with first dosing in rats pretreated with vehicle (Fig. 2, A and C; Rep. Veh-Veh versus Rep. Veh-Fasi). Unexpectedly, because of the increase in early-phase insulin response by vehicle treatment, the significant increase in postprandial insulin secretion in response to fasiglifam was not observed in rats treated with fasiglifam long term (Fig. 2, B and D). Immunostaining of pancreatic islets with anti-insulin and antiglucagon antibodies showed no apparent differences between the long-term vehicleand fasiglifam-treated groups (Fig. 3).

Fasiglifam Is Effective in Diabetic Rats with SU Secondary Failure. To confirm the effects of fasiglifam in a type 2 diabetic model with SU secondary failure, N-STZ-1.5 rats were given glibenclamide at $10 \mathrm{mg} / \mathrm{kg}$ per day for 4 weeks according to methods previously reported (Asakawa et al., 2009). In diabetic N-STZ-1.5 rats that were given vehicle for 4 weeks, single oral administration of glibenclamide $(10 \mathrm{mg} / \mathrm{kg}$ ) significantly improved glucose tolerance, with an increase in insulin secretion (Fig. 4; Rep. Veh-Veh versus Rep. Veh-Glib). In contrast, after 4 weeks of treatment with glibenclamide $(10 \mathrm{mg} / \mathrm{kg})$, glucose tolerance was exacerbated (Fig. 4, A and C; Rep. Veh-Veh versus Rep. GlibVeh), and the response to glibenclamide (10 $\mathrm{mg} / \mathrm{kg})$ was completely lost (Fig. 4; Rep. Glib-Veh versus Rep. Glib-Glib), which suggests that these rats developed SU secondary failure. A single dose of fasiglifam (3 mg/kg) significantly improved glucose tolerance with an increase in insulin secretion after treatment with glibenclamide (Fig. 4; Rep. Glib-Veh versus Rep. Glib-Fasi).

Fasiglifam Improves Glycemic Control in Severe Diabetic Rats Unresponsive to SUs. It was previously reported that long-term treatment with SUs are ineffective in lowering blood glucose levels in ZDF rats that develop overt diabetes due to gradually impaired $\beta$-cell function (Atkinson et al., 2008). To further evaluate the efficacy of fasiglifam in diabetic rats that are less responsive to SUs, we examined the glucose-lowering effects of fasiglifam in aged ZDF rats. Fasting plasma glucose levels were significantly elevated in 15-week-old ZDF rats compared with age-matched control rats (Fig. 5A; $367.5 \pm 58.0$ versus $103.8 \pm 4.2 \mathrm{mg} / \mathrm{dl}$ ), which suggests the development of severe diabetes in these rats. As expected, single administration of glimepiride $(10 \mathrm{mg} / \mathrm{kg})$ did not result in a significant decrease in fasting plasma glucose and increase in plasma insulin in these rats (Fig. 5, A and B). On the other hand, fasiglifam (10 mg/kg) significantly improved fasting hyperglycemia and enhanced insulin secretion in these rats (Fig. 5).

Combination of Fasiglifam and Glimepiride Additively Improves Glucose Tolerance in Diabetic Rats. We next evaluated combined effects of fasiglifam and glimepiride on glucose tolerance in N-STZ-1.5 rats. Compared with vehicle, oral administration of fasiglifam $(3 \mathrm{mg} / \mathrm{kg})$ or glimepiride (10 mg/kg) 1 hour before glucose load decreased plasma glucose AUC by 25.3 or $20.0 \%$, respectively; combination of fasiglifam and glimepiride resulted in a further decrease in plasma glucose AUC by $43.1 \%$ compared with either drug alone (Fig. 6, A and C). Two-way ANOVA showed that the combination had additive glucose-lowering effects (fasiglifam, $P \leq 0.01$; glimepiride, $P \leq 0.01$; interaction, not significant). In accordance with the glucose-lowering effects, fasiglifam, glimepiride, or the combination of these drugs enhanced insulin secretion 1.4-, 1.3-, or 1.7-fold, respectively, compared with vehicle (Fig. 6, B and D); the combined effect of the drugs was additive (fasiglifam, $P \leq 0.01$; glimepiride, $P \leq 0.05$; interaction, not significant by two-way ANOVA). In rats given combination treatment with fasiglifam and glimepiride, insulin secretion was restored to a level comparable to control rats (Fig. 6, B and D). These results indicate that fasiglifam and glimepiride improve hyperglycemia and enhance insulin release in a complementary manner. 
A
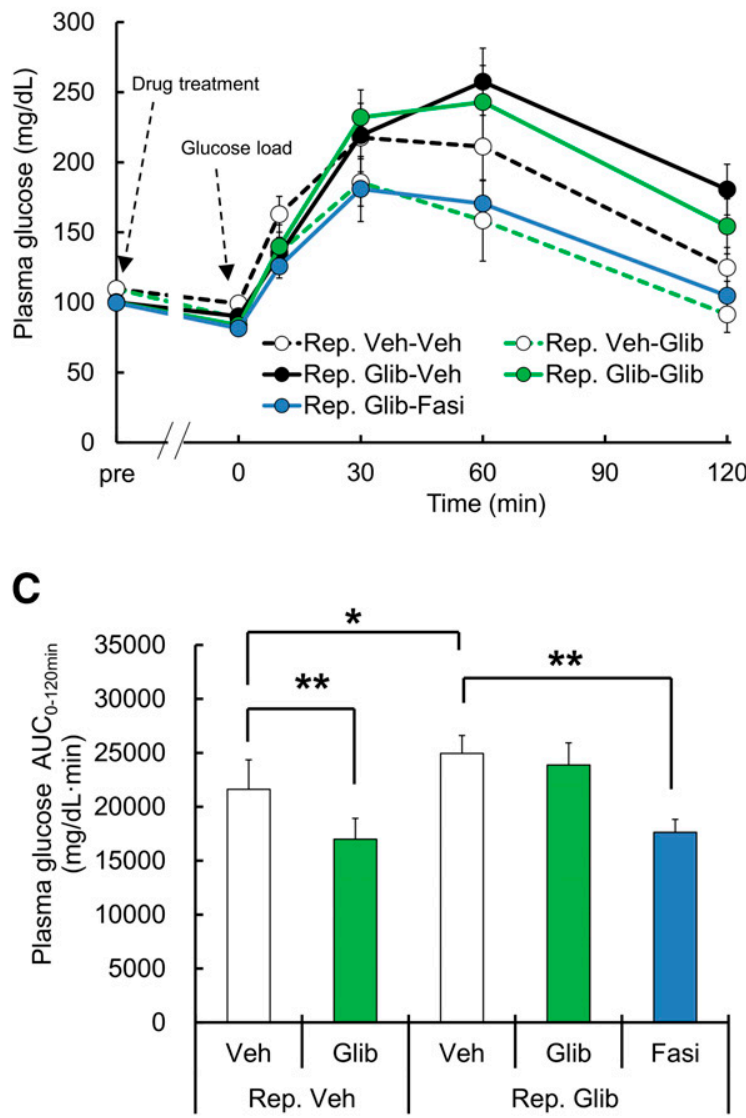

B

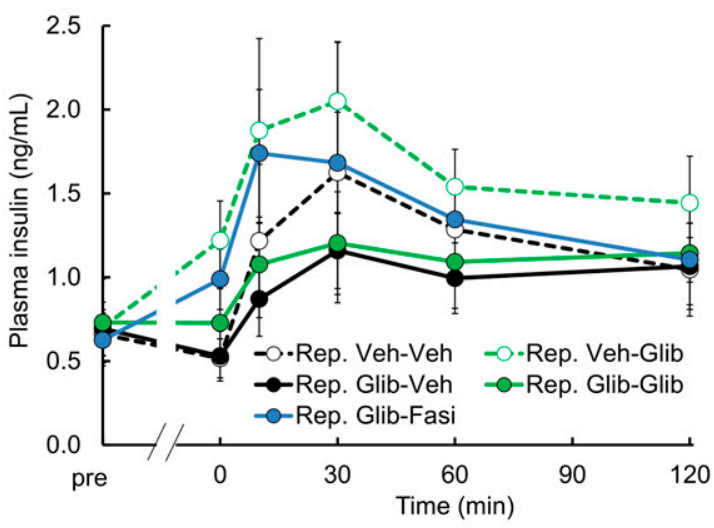

D

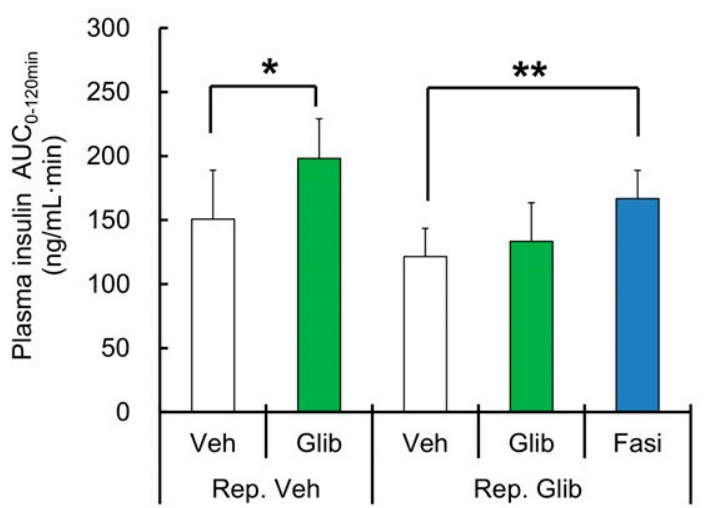

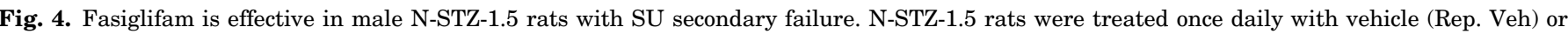

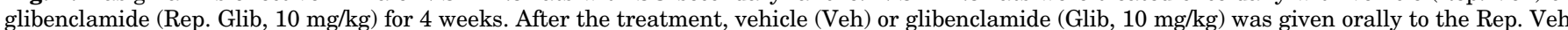

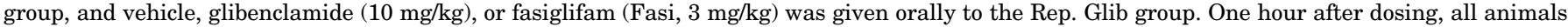

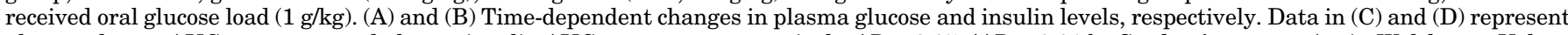

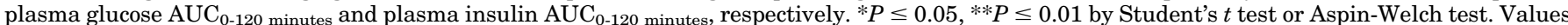
are mean \pm S.D. $(n=8)$.

Fasiglifam neither Induces Hypoglycemia nor Exacerbates SU-Induced Hypoglycemia in Normal Rats. A previous in vitro report has noted that glucose dependency for GPR40-mediated insulin secretion is uncoupled by the presence of SUs (Yang et al., 2010), which implies that fasiglifam would enhance in vivo insulin secretion independent of plasma glucose levels when administered concomitantly with SUs, thereby promoting hypoglycemia caused by SUs. Thus, we evaluated the risk of hypoglycemia in nonfasted normal Sprague-Dawley rats treated with combination of fasiglifam and glimepiride. Oral administration of glimepiride $(10 \mathrm{mg} / \mathrm{kg})$ lowered plasma glucose levels below $65 \mathrm{mg} / \mathrm{dl}$, leading to hypoglycemia (Fig. 7, A and C). Consistent with its glucoselowering effects, glimepiride significantly enhanced insulin secretion compared with vehicle, with a peak increase 60 minutes after dosing (Fig. 7, B and D). Fasiglifam ( $3 \mathrm{mg} / \mathrm{kg}$ ) had little effect on plasma glucose and insulin release (Fig. 7, $\mathrm{A}$ and $\mathrm{B}$ ), although slight but significant decrease in plasma glucose AUC was observed (Fig. 7C). Interestingly, when coadministered with fasiglifam $(3 \mathrm{mg} / \mathrm{kg}$ ) and glimepiride $(10 \mathrm{mg} / \mathrm{kg})$, there were neither further decreases in plasma glucose AUC nor further increases in plasma insulin AUC compared with glimepiride alone (Fig. 7, C and D). Under the same conditions, oral administration of the DPP-4 inhibitor alogliptin $(1 \mathrm{mg} / \mathrm{kg})$, a comparator that shows glucosedependent insulin release, also did not lower plasma glucose levels less than the normal range; in combination with glimepiride $(10 \mathrm{mg} / \mathrm{kg})$, the combination did not result in a further reduction of plasma glucose levels compared with glimepiride alone (Fig. 7, A and C). These results indicate that fasiglifam does not exacerbate SU-induced hypoglycemia in combination with SUs in normal rats and that the risk of hypoglycemia with fasiglifam and $\mathrm{SU}$ is not higher than combination treatment of a DPP4 inhibitor and SU.

\section{Discussion}

Fasiglifam, a potent and selective GPR40 agonist, provides glucose-dependent insulin secretion through activation of the G $\alpha$ q signaling pathway (Tsujihata et al., 2011), which is a mechanism distinct from other clinically available insulinotropic drugs (Winzell and Ahren, 2007). Fasiglifam shows glucose-lowering effects due to the augmentation of insulin secretion in type 2 diabetic rats (Tsujihata et al., 2011; Ito et al., 2013) and in patients with diabetes (Burant et al., 2012; Kaku et al., 2013). However, the clinical development of fasiglifam was terminated because of concerns about liver safety. This provides a key message: a deeper and more 


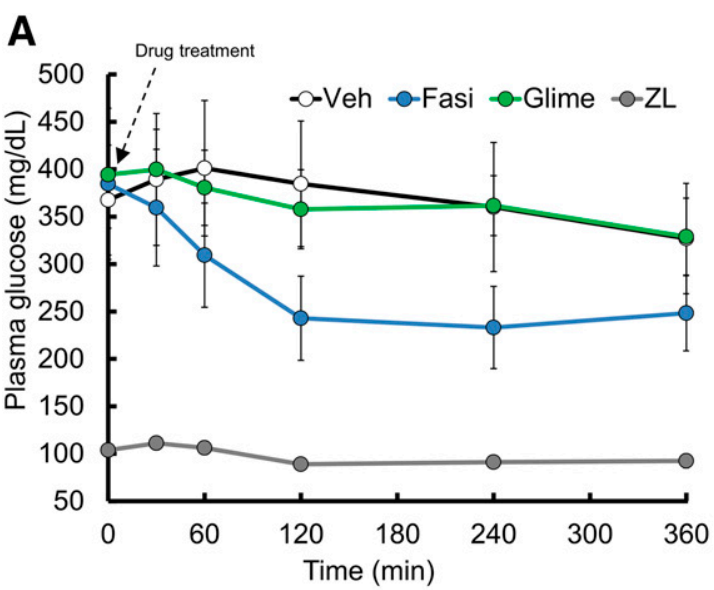

B

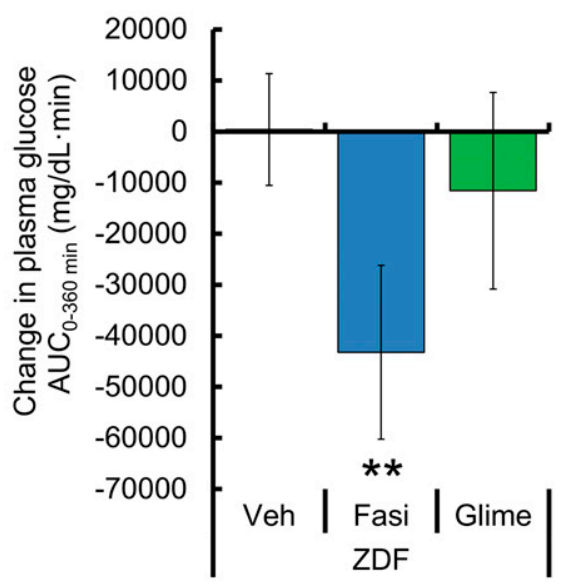

C

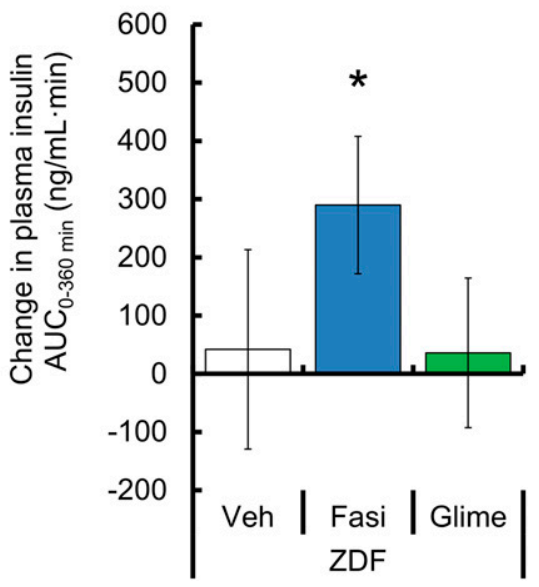

Fig. 5. Fasiglifam improves glycemic control in severe diabetic rats unresponsive to SU. (A) Time-dependent changes in plasma glucose levels in 15-week-old male ZDF and ZL rats (normal controls) after treatment with vehicle (Veh), $10 \mathrm{mg} / \mathrm{kg}$ of fasiglifam (Fasi), or $10 \mathrm{mg} / \mathrm{kg}$ of glimepiride (Glime). Data in (B) and $(C)$ represent change in plasma glucose $\mathrm{AUC}_{0-360 \text { minutes }}$ and change in plasma insulin $\mathrm{AUC}_{0-360}$ minutes after drug treatments, respectively. Values are mean \pm S.D. $(n=6)$. $* P \leq 0.05, * * P \leq 0.01$ compared with vehicle by Student's $t$ test or Aspin-Welch test. detailed understanding of its basic biology and pharmacology is required (Mancini and Poitout, 2015). Given in addition to fasiglifam, several other GPR40 agonists are currently still in the preclinical and clinical stages of development (Mancini and Poitout, 2015), GPR40 agonists potentially constitute a new class of antidiabetic drugs. Thus, there is a need to examine their differences from and interactions with widely used insulinotropic drugs. Although comparison of in vitro profiles between GPR40 agonist and SUs have been reported thus far (Zhao et al., 2008; Yang et al., 2010; Tsujihata et al., 2011), the differences in in vivo acute and chronic efficacies between these drugs in diabetic rodents are not yet fully understood. It is also unclear whether these drugs could work cooperatively. In the present study, our data indicated that fasiglifam improved postprandial hyperglycemia more effectively than glibenclamide. Our results showed that chronic GPR40 activation by fasiglifam did not cause secondary loss of the glucose-lowering efficacy, in contrast to glibenclamide, indicating that GPR40 agonists have the better durability compared with SUs. Interestingly, fasiglifam was still effective in two different rodent models that were completely desensitized to SUs. In addition, fasiglifam improved glucose excursion and augmented insulin secretion additively with glimepiride in N-STZ-1.5 rats and did not exacerbate glimepiride-induced hypoglycemia in control rats. These observations suggest that GPR40 agonists could be broadly useful as glycemic rescue agents in patients who have already undergone treatment with SUs.
In the present study, compared at a maximal dose of $30 \mathrm{mg} / \mathrm{kg}$, the glucose-lowering effects and insulinotropic action of fasiglifam after glucose load were significantly greater than those of glibenclamide. Of note, at these doses, the effects on fasting plasma glucose and insulin levels before glucose load were not different between drugs, indicating that fasiglifam exerts robust effects on insulin release especially under the postprandial condition. It is likely that in the postprandial condition, $\mathrm{K}_{\mathrm{ATP}}$ channel activity in $\beta$-cells is already potently inhibited by high glucose, which may preclude the further inhibitory effect of glibenclamide. Another possible explanation is that the interaction of fasiglifam with the gut hormones released from enteroendocrine cells might contribute to the robust postprandial insulin release by fasiglifam treatment in our study. It has been reported that glucagon-like peptide-1 and acetylcholine, which activates the same G $\alpha$ q signaling pathway as GPR40 agonists, exert synergistic effects on insulin secretion in perfused rat islets (Zawalich et al., 1993). In future study, the mechanisms by which these gut hormones interact with GPR40 agonists and SUs should be assessed.

Many G-protein-coupled receptors involved in insulin secretion in $\beta$-cells are known to undergo in vitro rapid desensitization subsequent to repeated stimulation by those specific agonists (DeRubertis and Craven, 1976; Hinke et al., 2000; Baggio et al., 2004). Moreover, because chronic exposure of FFAs, endogenous ligands for GPR40, causes the harmful 
A

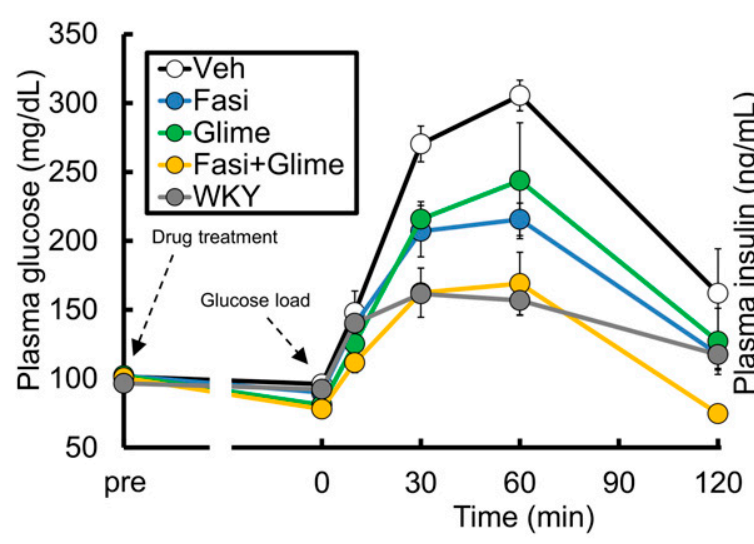

C

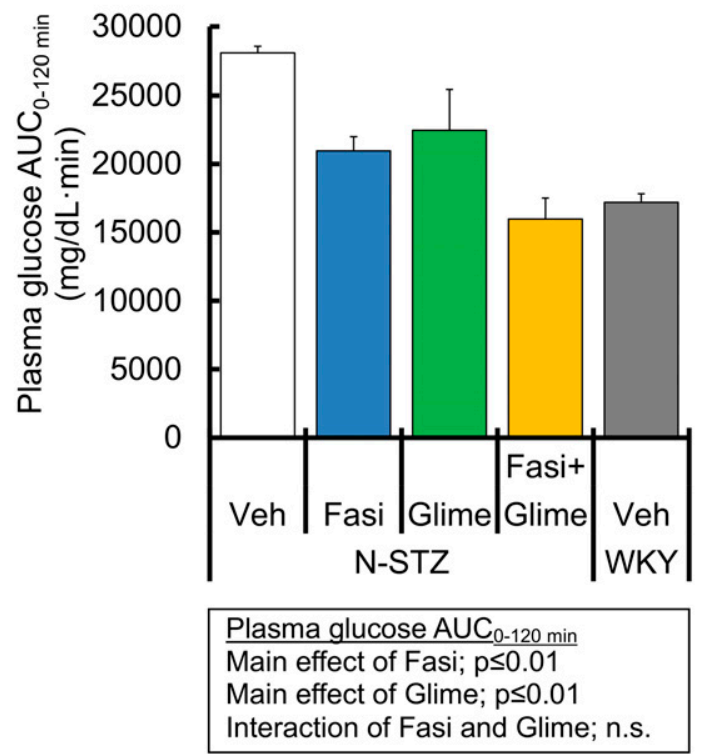

B

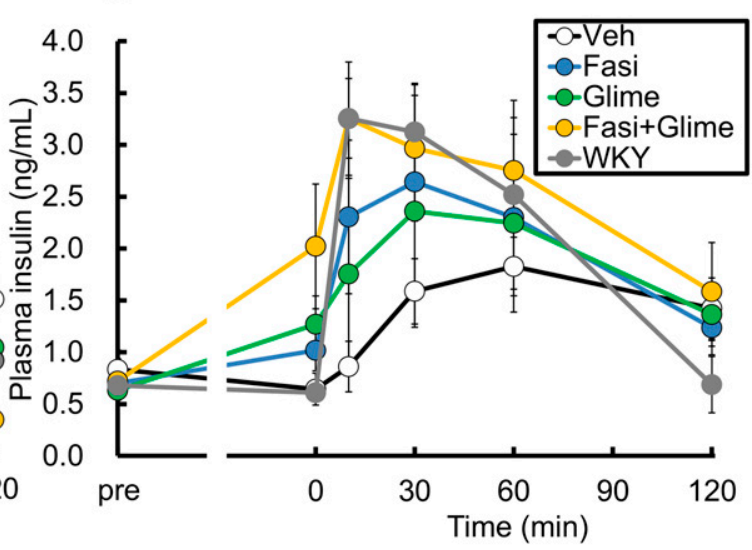

\section{D}

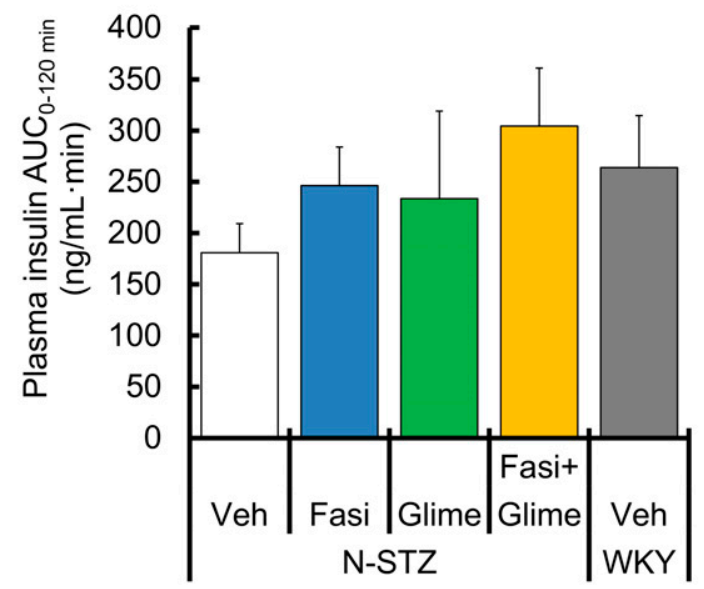

\begin{tabular}{|l|}
\hline Plasma insulin AUC \\
Main effect of Fasi; $p \leq 0.01$ \\
Main effect of Glime; $p \leq 0.05$ \\
Interaction of Fasi and Glime; $n$.s.
\end{tabular}

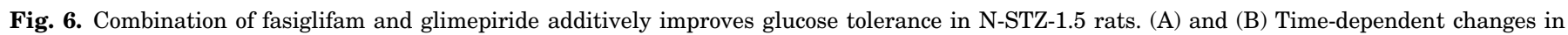

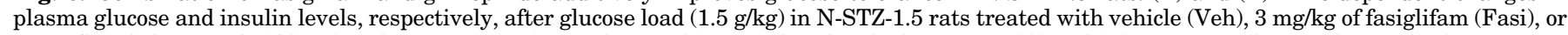

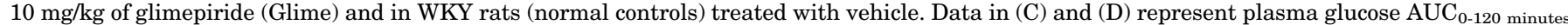

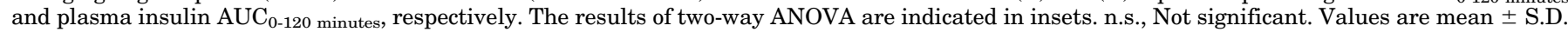
( $n=6$ for N-STZ-1.5 rats, $n=5$ for WKY rats).

effects on $\beta$-cell viability (lipotoxicity), there is great interest in the impact after long-term activation of GPR40 by selective agonists. In this study, in contrast to glibenclamide, even after almost 4 months of treatment in N-STZ-1.5 rats, the glucoselowering effects of fasiglifam were well preserved to a level comparable to that of the first administration, which is consistent with several short-term studies with other GPR40 agonists (Tan et al., 2008; Lin et al., 2011). These results indicate that GPR40 agonists may have a low risk of receptor desensitization. Furthermore, in our study, there were no apparent differences in islet morphology between the vehicleand fasiglifam-treated groups. Several experimental observations from genetically modified mice suggest that GPR40 is less involved in lipotoxicity (Latour et al., 2007; Kebede et al., 2008; Nagasumi et al., 2009). In addition, fasiglifam significantly improves glycemic control and is well tolerated after 24 weeks of treatment in patients with type 2 diabetes (Kaku et al., 2013). These findings support that chronic exposure to
GPR40 agonist does not cause $\beta$-cell dysfunction. Regarding the low risk of receptor desensitization, it might be explained by the unique binding profile of fasiglifam. It was recently demonstrated that fasiglifam is an ago-allosteric modulator of GPR40, which binds to a different binding site from the orthosteric site (Yabuki et al., 2013; Srivastava et al., 2014). Because it has been shown that some allosteric modulators allow receptor activation with less propensity for receptor desensitization than orthosteric agonists (Gjoni and Urwyler, 2008), it is speculated that fasiglifam may show the similar profile.

After long-term SU treatment, some patients begin to suffer from the secondary failure (Matthews et al., 1998). There are several factors that are involved in the development of SU secondary failure, including $\beta$-cell exhaustion by long-term overstimulation and glucose toxicity caused by poor glycemic control (Poitout and Robertson, 2002; Rustenbeck, 2002). In our study, N-STZ-1.5 rats receiving glibenclamide for 4 weeks 
A

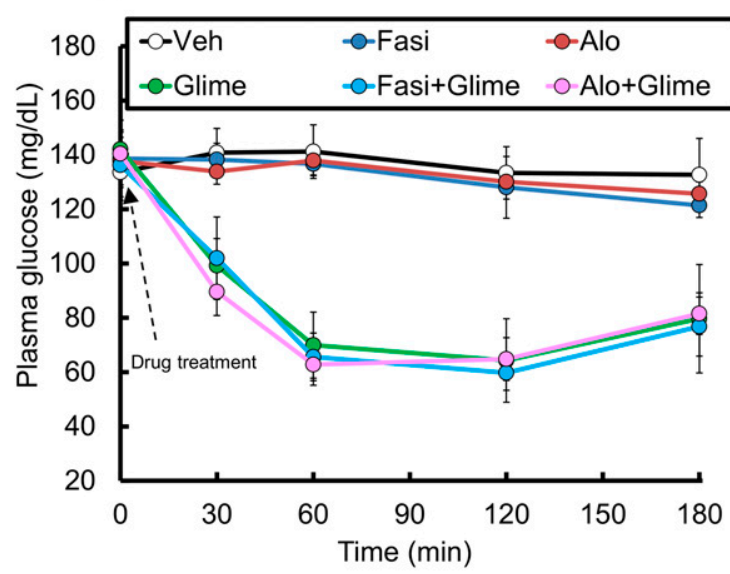

C

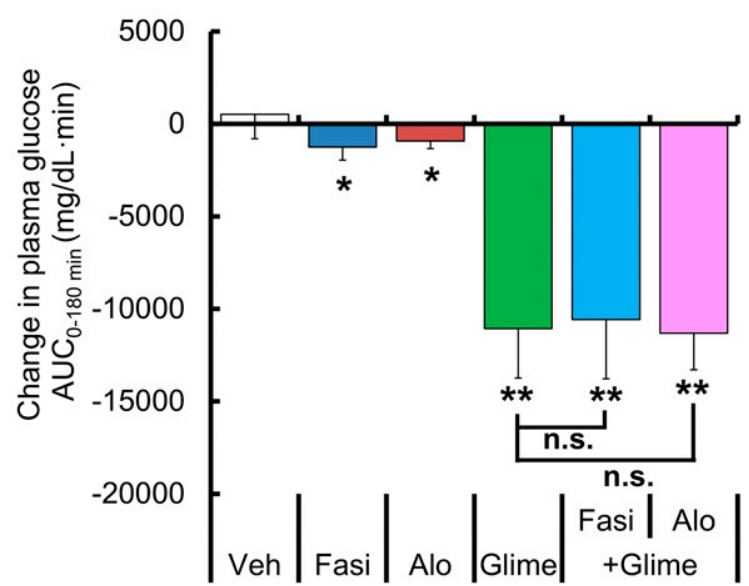

B

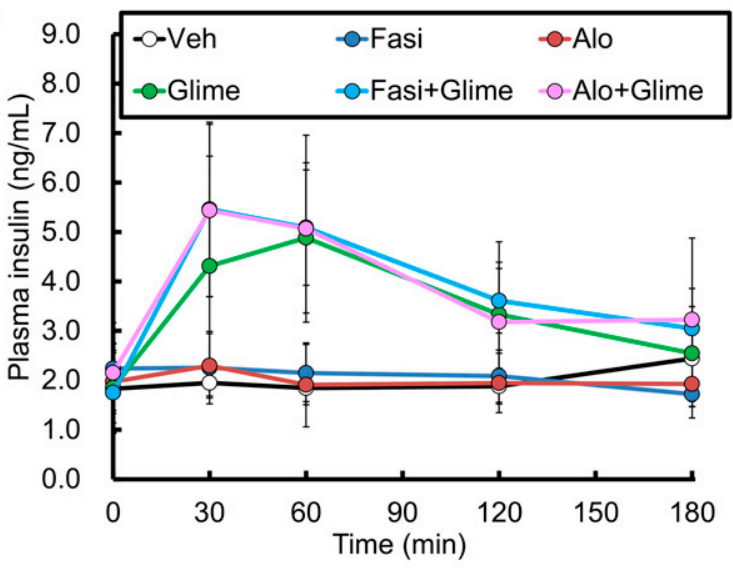

D

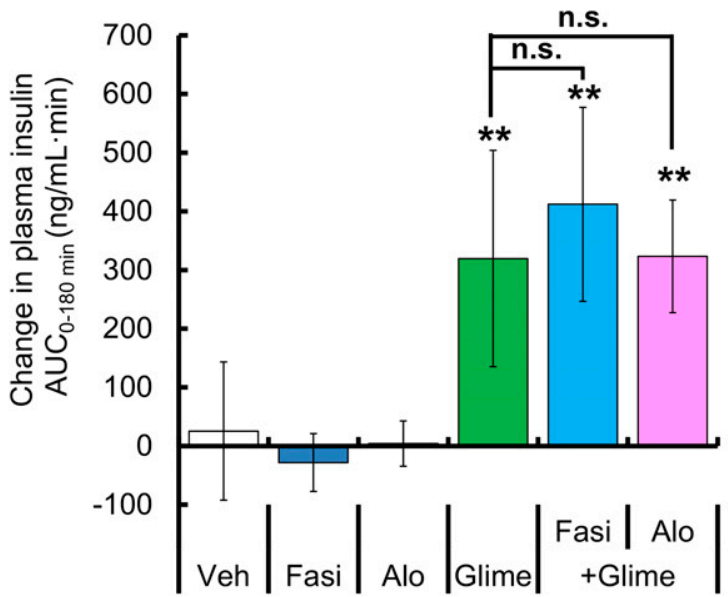

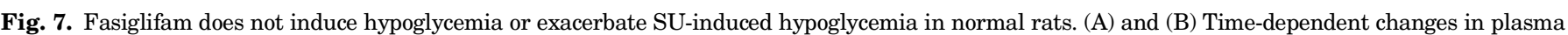

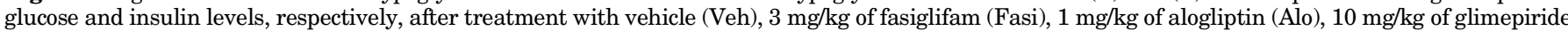

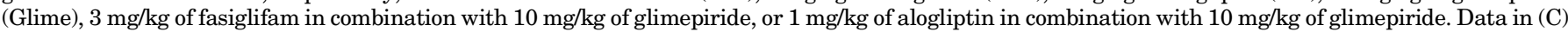

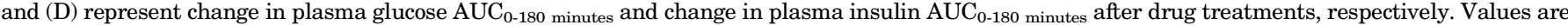
mean \pm S.D. $(n=6)$. $* P \leq 0.05, * * P \leq 0.01$ compared with vehicle by Student's $t$ test or Aspin-Welch test. n.s., Not significant.

exhibited an exacerbation of glucose tolerance and a loss in response to glibenclamide. Considering that these rats exhibited normal fasting plasma glucose levels, the unresponsiveness of $\beta$-cells to SUs noted in this model may be mainly due to desensitization of insulin secretion machinery $(\beta$-cell exhaustion) rather than glucose toxicity. The previous in vitro study indicated that $\beta$-cells chronically treated with glibenclamide show reduced functional $\mathrm{K}_{\mathrm{ATP}}$ channels on plasma membranes, despite elevated intracellular $\mathrm{Ca}^{2+}$ (Anello et al., 1999; Kawaki et al., 1999). In the present study, fasiglifam successfully enhanced insulin release in rats after 4 weeks of treatment with glibenclamide. This observation implies that functional $\mathrm{K}_{\mathrm{ATP}}$ channels may not always be required for triggering insulin secretion via GPR40 activation under the presence of enough $\mathrm{Ca}^{2+}$. Consistent with our finding, it has been shown that GPR40 agonists increase insulin secretion caused by voltage-dependent $\mathrm{Ca}^{2+}$ channel activators, which induces direct influx of $\mathrm{Ca}^{2+}$, not via $\mathrm{K}_{\mathrm{ATP}}$ channels (Yang et al., 2010).

In the present study, the unresponsiveness of $\beta$-cells to glimepiride was also observed in aged ZDF rats. This result is inconsistent with the previous observation that glibenclamide shows a trend toward improved fasting hyperglycemia in ZDF rats (Tsujihata et al., 2011). The discrepancy may be due to the differences in disease severity and age of the rats used in the two studies. In contrast to glibenclamide-treated N-STZ-1.5 rats, hyperglycemia per se likely contributes to SU desensitization in ZDF rats, probably through increased $\beta$-cell death, decreased insulin content, and decreased glucose-sensing genes (Lee et al., 1994; Tokuyama et al., 1995; Harmon et al., 2001). Interestingly, single administration of fasiglifam also robustly increased insulin secretion and improved glycemic control in this model, suggesting the GPR40 and the downstream signaling molecules are sufficiently maintained even in severe diabetic rodents with $\beta$-cell dysfunction.

It was previously reported that glucose dependency for GPR40-mediated insulin secretion is uncoupled by the presence of SUs in rat insulinoma cells (Yang et al., 2010), which implies that GPR40 agonists would enhance insulin secretion even at low plasma glucose levels when administered with SUs. In our study, fasiglifam in combination with glimepiride showed an additive effect in the improvement of glucose excursion and augmentation of insulin secretion. Conversely, when administered together at the same doses 
in normal rats, fasiglifam did not exacerbate glimepirideinduced hypoglycemia and further increase plasma insulin levels. These results suggest that uncoupling of glucosedependent effects of fasiglifam may not occur so much in vivo in the presence of SUs. Insulin secretion is intricately regulated by several factors other than glucose, which likely contribute in part to the different observations noted in in vitro and in vivo studies. In the present study, a DPP-4 inhibitor, alogliptin, did not also enhance glimepiride-induced hypoglycemia in normal rats. However, there are a few cases of severe hypoglycemia with DPP-4 inhibitor and SU combination in elderly and/or patients with renal insufficiency when relatively higher dose of SUs are administered (Yabe and Seino, 2014). Although it has been reported that fasiglifam alone has very low hypoglycemia risk in the clinical trials (Burant et al., 2012), the reported results of DPP-4 inhibitor and SU combination suggest that careful monitoring should be required in humans especially in the combination therapy with GPR40 agonists and SUs.

In conclusion, the present study clearly indicates that compared with SUs, the GPR40 agonists are effective in lowering glucose, with less risk of secondary failure and hypoglycemia. Moreover, our findings could provide further insights into the pharmacology of GPR40 agonists and the rationale for their use in patients with diabetes with SU secondary failure. GPR40 agonists could also provide further improvement of glycemic control even when used in patients already receiving SU therapy; however, further investigation regarding the clinical risk of hypoglycemia is warranted.

\section{Acknowledgments}

The authors thank Drs. Seigo Izumo, Yukio Yamada, Masakuni Noda, and Nobuhiro Nishigaki for offering valuable discussions and helpful suggestions; Hirohisa Miyashita for providing helpful support; and Toshikazu Ando and Shoichi Asano for technical assistance.

\section{Authorship Contributions}

Participated in research design: Ito, Tsujihata, Suzuki, Miyawaki, Matsuda, and Takeuchi.

Conducted experiments: Ito, Tsujihata, Suzuki, Miyawaki, Matsuda, and Takeuchi.

Performed data analysis: Ito, Tsujihata, Suzuki, and Miyawaki.

Wrote or contributed to the writing of the manuscript: Ito, Tsujihata, and Takeuchi.

\section{References}

Anello M, Gilon P, and Henquin JC (1999) Alterations of insulin secretion from mouse islets treated with sulphonylureas: perturbations of $\mathrm{Ca} 2+$ regulation prevail over changes in insulin content. Br J Pharmacol 127:1883-1891.

Asakawa T, Moritoh Y, Kataoka O, Suzuki N, Takeuchi K, and Odaka H (2009) A novel dipeptidyl peptidase-4 inhibitor, alogliptin (SYR-322), is effective in diabetic rats with sulfonylurea-induced secondary failure. Life Sci 85:122-126.

Atkinson LL, McDonald-Dyck C, Benkoczi C, and Finegood DT (2008) Effect of chronic rosiglitazone, metformin and glyburide treatment on beta-cell mass, function and insulin sensitivity in mZDF rats. Diabetes Obes Metab 10:780-790.

Baggio LL, Kim JG, and Drucker DJ (2004) Chronic exposure to GLP-1R agonists promotes homologous GLP-1 receptor desensitization in vitro but does not attenuate GLP-1R-dependent glucose homeostasis in vivo. Diabetes 53 (Suppl 3): S205-S214.

Briscoe CP, Tadayyon M, Andrews JL, Benson WG, Chambers JK, Eilert MM, Ellis C, Elshourbagy NA, Goetz AS, and Minnick DT, et al. (2003) The orphan G proteincoupled receptor GPR40 is activated by medium and long chain fatty acids. J Biol Chem 278:11303-11311.

Burant CF, Viswanathan P, Marcinak J, Cao C, Vakilynejad M, Xie B, and Leifke E (2012) TAK-875 versus placebo or glimepiride in type 2 diabetes mellitus: a phase 2, randomised, double-blind, placebo-controlled trial. Lancet 379:1403-1411.

Charpentier G (2002) Oral combination therapy for type 2 diabetes. Diabetes Metab Res Rev 18 (Suppl 3):S70-S76.

DeRubertis FR and Craven P (1976) Effects of reduced ATP concent on hepatic responses to glucagon. Metabolism 25:57-67.
Drucker DJ (2007) Dipeptidyl peptidase-4 inhibition and the treatment of type 2 diabetes: preclinical biology and mechanisms of action. Diabetes Care 30: 1335-1343.

Feng DD, Luo Z, Roh SG, Hernandez M, Tawadros N, Keating DJ, and Chen C (2006) Reduction in voltage-gated $\mathrm{K}+$ currents in primary cultured rat pancreatic betacells by linoleic acids. Endocrinology 147:674-682.

Feng J, Zhang Z, Wallace MB, Stafford JA, Kaldor SW, Kassel DB, Navre M, Shi L, Skene RJ, and Asakawa T, et al. (2007) Discovery of alogliptin: a potent, selective, bioavailable, and efficacious inhibitor of dipeptidyl peptidase IV. J Med Chem 50: $2297-2300$

Fujiwara K, Maekawa F, and Yada T (2005) Oleic acid interacts with GPR40 to induce $\mathrm{Ca} 2+$ signaling in rat islet beta-cells: mediation by PLC and L-type Ca2+ channel and link to insulin release. Am J Physiol Endocrinol Metab 289: E670-E677.

Gjoni T and Urwyler S (2008) Receptor activation involving positive allosteric modulation, unlike full agonism, does not result in GABAB receptor desensitization. Neuropharmacology 55:1293-1299.

Harmon JS, Gleason CE, Tanaka Y, Poitout V, and Robertson RP (2001) Antecedent hyperglycemia, not hyperlipidemia, is associated with increased islet triacylglycerol content and decreased insulin gene mRNA level in Zucker diabetic fatty rats. Diabetes 50:2481-2486.

Hinke SA, Pauly RP, Ehses J, Kerridge P, Demuth HU, McIntosh CH, and Pederson RA (2000) Role of glucose in chronic desensitization of isolated rat islets and mouse insulinoma (betaTC-3) cells to glucose-dependent insulinotropic polypeptide. J Endocrinol 165:281-291.

Ito R, Tsujihata Y, Matsuda-Nagasumi K, Mori I, Negoro N, and Takeuchi K (2013) TAK-875, a GPR40/FFAR1 agonist, in combination with metformin prevents progression of diabetes and $\beta$-cell dysfunction in Zucker diabetic fatty rats. $\mathrm{Br}$ $J$ Pharmacol 170:568-580.

Itoh Y, Kawamata Y, Harada M, Kobayashi M, Fujii R, Fukusumi S, Ogi K, Hosoya M, Tanaka Y, and Uejima H, et al. (2003) Free fatty acids regulate insulin secretion from pancreatic beta cells through GPR40. Nature 422:173-176.

Kaku K, Araki T, and Yoshinaka R (2013) Randomized, double-blind, dose-ranging study of TAK-875, a novel GPR40 agonist, in Japanese patients with inadequately controlled type 2 diabetes. Diabetes Care 36:245-250.

Kaku K, Enya K, Nakaya R, Ohira T, and Matsuno R (2015) Efficacy and safety of fasiglifam (TAK-875), a G protein-coupled receptor 40 agonist, in Japanese patients with type 2 diabetes inadequately controlled by diet and exercise: a randomized, double-blind, placebo-controlled, phase III trial. Diabetes Obes Metab 17: 675-681.

Kawaki J, Nagashima K, Tanaka J, Miki T, Miyazaki M, Gonoi T, Mitsuhashi N, Nakajima N, Iwanaga T, and Yano H, et al. (1999) Unresponsiveness to glibenclamide during chronic treatment induced by reduction of ATP-sensitive $\mathrm{K}+$ channel activity. Diabetes 48:2001-2006.

Kebede M, Alquier T, Latour MG, Semache M, Tremblay C, and Poitout V (2008) The fatty acid receptor GPR40 plays a role in insulin secretion in vivo after high-fat feeding. Diabetes 57:2432-2437.

Kotarsky K, Nilsson NE, Flodgren E, Owman C, and Olde B (2003) A human cell surface receptor activated by free fatty acids and thiazolidinedione drugs. Biochem Biophys Res Commun 301:406-410.

Latour MG, Alquier T, Oseid E, Tremblay C, Jetton TL, Luo J, Lin DC, and Poitout V (2007) GPR40 is necessary but not sufficient for fatty acid stimulation of insulin secretion in vivo. Diabetes 56:1087-1094.

Leahy JL (2005) Pathogenesis of type 2 diabetes mellitus. Arch Med Res 36: 197-209.

Lee Y, Hirose H, Ohneda M, Johnson JH, McGarry JD, and Unger RH (1994) Betacell lipotoxicity in the pathogenesis of non-insulin-dependent diabetes mellitus of obese rats: impairment in adipocyte-beta-cell relationships. Proc Natl Acad Sci USA 91:10878-10882.

Lin DC, Zhang J, Zhuang R, Li F, Nguyen K, Chen M, Tran T, Lopez E, Lu JY, and Li $\mathrm{XN}$, et al. (2011) AMG 837: a novel GPR40/FFA1 agonist that enhances insulin secretion and lowers glucose levels in rodents. PLoS One 6:e27270.

Mancini AD and Poitout V (2015) GPR40 agonists for the treatment of type 2 diabetes: life after 'TAKing' a hit. Diabetes Obes Metab 17:622-629.

Matthews DR, Cull CA, Stratton IM, Holman RR, and Turner RC; UK Prospective Diabetes Study (UKPDS) Group (1998) UKPDS 26: Sulphonylurea failure in noninsulin-dependent diabetic patients over six years. Diabet Med 15:297-303.

Moritoh Y, Takeuchi K, Asakawa T, Kataoka O, and Odaka H (2009) Combining a dipeptidyl peptidase- 4 inhibitor, alogliptin, with pioglitazone improves glycaemic control, lipid profiles and beta-cell function in db/db mice. $B r J$ Pharmacol 157: $415-426$.

Nagasumi K, Esaki R, Iwachidow K, Yasuhara Y, Ogi K, Tanaka H, Nakata M, Yano T, Shimakawa K, and Taketomi S, et al. (2009) Overexpression of GPR40 in pancreatic beta-cells augments glucose-stimulated insulin secretion and improves glucose tolerance in normal and diabetic mice. Diabetes 58:1067-1076.

Naik H, Vakilynejad M, Wu J, Viswanathan P, Dote N, Higuchi T, and Leifke E (2012) Safety, tolerability, pharmacokinetics, and pharmacodynamic properties of the GPR40 agonist TAK-875: results from a double-blind, placebo-controlled single oral dose rising study in healthy volunteers. J Clin Pharmacol 52:1007-1016.

Negoro N, Sasaki S, Mikami S, Ito M, Suzuki M, Tsujihata Y, Ito R, Harada A Takeuchi K, and Suzuki N, et al. (2010) Discovery of TAK-875: a potent, selective, and orally bioavailable GPR40 agonist. ACS Med Chem Lett 1:290-294.

Negoro N, Sasaki S, Mikami S, Ito M, Tsujihata Y, Ito R, Suzuki M, Takeuchi K, Suzuki N, and Miyazaki J, et al. (2012) Optimization of (2,3-dihydro-1-benzofuran3-yl)acetic acids: discovery of a non-free fatty acid-like, highly bioavailable G protein-coupled receptor $40 /$ free fatty acid receptor 1 agonist as a glucosedependent insulinotropic agent. J Med Chem 55:3960-3974.

Poitout V and Robertson RP (2002) Minireview: Secondary beta-cell failure in type 2 diabetes-a convergence of glucotoxicity and lipotoxicity. Endocrinology 143:339-342. 
Portha B, Blondel O, Serradas P, McEvoy R, Giroix MH, Kergoat M, and Bailbe D (1989) The rat models of non-insulin dependent diabetes induced by neonatal streptozotocin. Diabete Metab 15:61-75.

Rendell M (2004) The role of sulphonylureas in the management of type 2 diabetes mellitus. Drugs 64:1339-1358.

Rustenbeck I (2002) Desensitization of insulin secretion. Biochem Pharmacol 63 . 1921-1935.

Sakuma K, Yabuki C, Maruyama M, Abiru A, Komatsu H, Negoro N, Tsujihata Y, Takeuchi K, Habata Y, and Mori M (2015) The individual roles of inositol triphosphate and diacylglycerol, downstream messengers of FFAR1/GPR40 produced by fasiglifam on the glucose-dependent insulinotropic effect. Diabetes 64 (Suppl 1): A574-A602.

Shapiro H, Shachar S, Sekler I, Hershfinkel M, and Walker MD (2005) Role of GPR40 in fatty acid action on the beta cell line INS-1E. Biochem Biophys Res Commun 335:97-104.

Srivastava A, Yano J, Hirozane Y, Kefala G, Gruswitz F, Snell G, Lane W, Ivetac A, Aertgeerts K, and Nguyen J, et al. (2014) High-resolution structure of the human GPR40 receptor bound to allosteric agonist TAK-875. Nature $\mathbf{5 1 3}$ 124-127.

Tan CP, Feng Y, Zhou YP, Eiermann GJ, Petrov A, Zhou C, Lin S, Salituro G, Meinke P, and Mosley R, et al. (2008) Selective small-molecule agonists of G proteincoupled receptor 40 promote glucose-dependent insulin secretion and reduce blood glucose in mice. Diabetes 57:2211-2219.

Tokuyama Y, Sturis J, DePaoli AM, Takeda J, Stoffel M, Tang J, Sun X, Polonsky KS, and Bell GI (1995) Evolution of beta-cell dysfunction in the male Zucker diabetic fatty rat. Diabetes 44:1447-1457.

Tsujihata Y, Ito R, Suzuki M, Harada A, Negoro N, Yasuma T, Momose Y, and Takeuchi K (2011) TAK-875, an orally available G protein-coupled receptor 40/free fatty acid receptor 1 agonist, enhances glucose-dependent insulin secretion and improves both postprandial and fasting hyperglycemia in type 2 diabetic rats. J Pharmacol Exp Ther 339:228-237.

Wajchenberg BL (2007) beta-cell failure in diabetes and preservation by clinical treatment. Endocr Rev 28:187-218.

Winzell MS and Ahrén B (2007) G-protein-coupled receptors and islet functionimplications for treatment of type 2 diabetes. Pharmacol Ther 116:437-448.

Yabe D and Seino Y (2014) Dipeptidyl peptidase-4 inhibitors and sulfonylureas for type 2 diabetes: Friend or foe? J Diabetes Investig 5:475-477.

Yabuki C, Komatsu H, Tsujihata Y, Maeda R, Ito R, Matsuda-Nagasumi K, Sakuma K, Miyawaki K, Kikuchi N, and Takeuchi K, et al. (2013) A novel antidiabetic drug, fasiglifam/TAK-875, acts as an ago-allosteric modulator of FFAR1. PLoS One 8: e76280.

Yang M, Chisholm JW, Soelaiman S, and Shryock JC (2010) Sulfonylureas uncouple glucose-dependence for GPR40-mediated enhancement of insulin secretion from INS-1E cells. Mol Cell Endocrinol 315:308-313.

Zawalich WS, Zawalich KC, and Rasmussen H (1993) Influence of glucagon-like peptide-1 on beta cell responsiveness. Regul Pept 44:277-283.

Zhao YF, Pei J, and Chen C (2008) Activation of ATP-sensitive potassium channels in rat pancreatic beta-cells by linoleic acid through both intracellular metabolites and membrane receptor signalling pathway. J Endocrinol 198:533-540.

Address correspondence to: Ryo Ito, Cardiovascular and Metabolic Drug Discovery Unit, Pharmaceutical Research Division, Takeda Pharmaceutical Company Limited, 26-1 Muraoka-higashi 2-Chome, Fujisawa, Kanagawa, 2518555, Japan. E-mail: ryo.ito@takeda.com 